Appl. Phys. 14, 149-153 (1977)

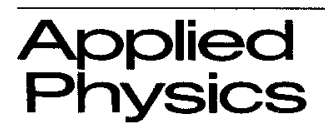

(C) by Springer-Verlag 1977

\title{
Field Emission of Monoenergetic Spin-Polarized Electrons
}

\author{
G. Baum, E. Kisker, A. H. Mahan ${ }^{\star}$, W. Raith, and B. Reihl \\ Fakultät für Physik, Universität, D-4800 Bielefeld, Fed. Rep. Germany
}

Received 2 May 1977/Accepted 13 June 1977

\begin{abstract}
Field emission from a tungsten tip, covered by a layer of crystallized ferromagnetic europium sulfide, leads to an electron beam with a current of $10^{-8} \mathrm{~A}$, an energy width of less than $100 \mathrm{meV}$, and a spin polarization of about 0.85 at a tip temperature of $9 \mathrm{~K}$. Proper annealing of the EuS layer is crucial.
\end{abstract}

PACS Codes: $79.40,75.30,72.20$

Polarized electron beams can be produced in various ways $[1,2]$, but none of the existing polarized electron sources combines the advantages of a very small physical size, a high polarization, and a small energy width. A field-emission source is an almost ideal point source well suited electron-optically for experiments with low-energy electrons. Highly polarized electrons can be obtained from W-EuS field emitters [3] at low temperatures. Studies of the W-EuS emission current and the electron spin polarization as a function of the tip temperature showed [4] that electrons tunnel from the Fermi level of tungsten into the EuS conduction band, as illustrated in Fig. 1. The internal barrier at the W-EuS boundary acts as a spin filter below the Curie temperature of EuS ( $T_{c}=16.5 \mathrm{~K}$ for pure crystalline samples), since the ferromagnetic band splitting of the EuS conduction band lowers (raises) the barrier for electrons with spins parallel (antiparallel) to the spins of the ferromagnetic $4 f^{7}$ electrons of the $\mathrm{Eu}^{++}$ions in the EuS crystal. The external barrier, lowered by the image potential, lies well below the Fermi level of tungsten and does not control the emission process.

During the last year we investigated the energy distribution of electrons from W-EuS emitters after annealing at different temperatures and found that the

* Present address: Physics Department, Rice University, Houston, TX 77001, USA emitted electrons are not monoenergetic $[5,6]$. In general, the energy distribution is broad, and extends to energies several electronvolts below the Fermi level of tungsten. In most cases we found a two-peak structure, as shown in Fig. 2. Recently, however, we discovered a unique condition of the W-EuS emitter, obtained after very specific annealing, which yields a highly polarized electron beam with a narrow energy distribution.

\section{Experiment}

The apparatus for field emission and Mott scattering polarization analysis is the same as that used in previous work [4].

The support of the W-EuS emitter is the most delicate part of the experimental set-up. Several designs were used in the course of this research program. The latest version is shown in Fig. 3. The helium-flow cooling system has been adapted from Reed and Graham [7]. The EuS is evaporated in situ from a tungsten oven which can be moved in front of the tip. Annealing of the EuS layer is done at a chosen temperature for several seconds by direct heating of the tungsten bow to which the tip is spot-welded. The annealing temperature is determined from the temperature-dependent resistance of the tungsten bow, given by the ratio of the potential drop across the bow to the heating current. The temperature is 


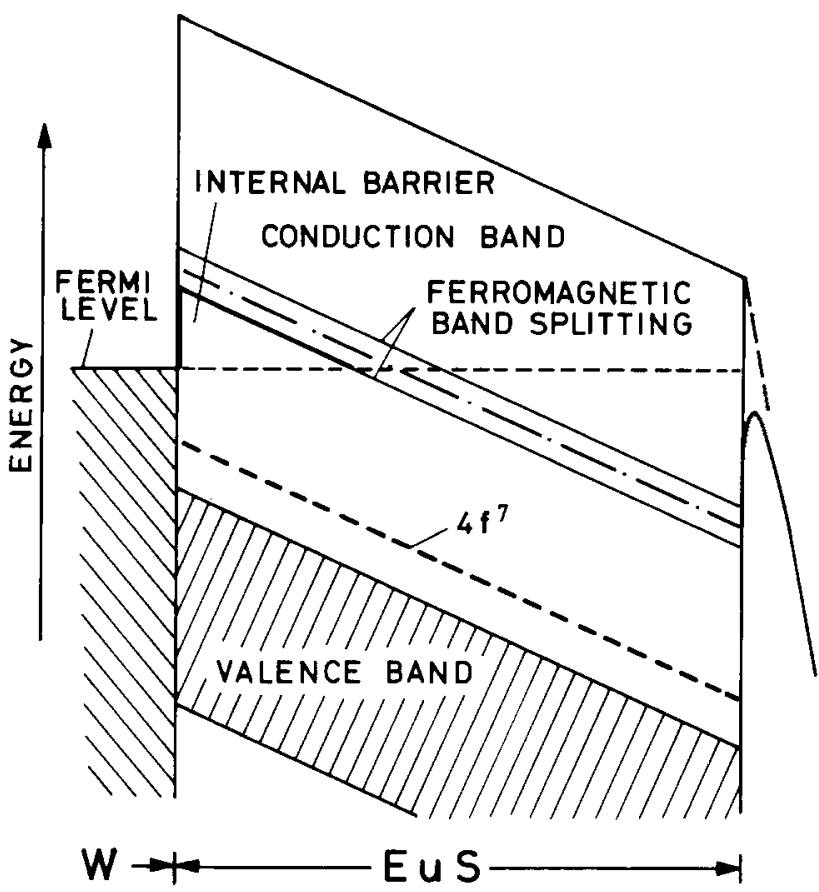

Fig. 1. Simplified band model for the W-EuS emitter. The applied external electric field penetrates into the insulating EuS layer and causes the band inclination; the internal field strength is reduced by the factor $1 / \varepsilon$, where $\varepsilon=10.2$ is the static dielectric constant of EuS. The emission current is governed by the internal barrier which is of different heights for the two spin states due to the ferromagnetic splitting of the EuS conduction band

kept constant by electronic regulation of the heating current.

The tungsten tip has a radius of about $1000 \AA$ and the $\langle 111\rangle$ direction along the tip axis [8]. A weak longitudinal magnetic field of 50 Gauss is used to produce a small image of the field-emission pattern which is viewed on a fluorescent screen. The screen has a "probe hole" of $2 \mathrm{~mm}$ diameter in its center; by means of electrostatic deflection a portion of the pattern is moved onto the hole for measuring both the spin polarization and the energy distribution of the selected electrons.

The polarization component transverse to the beam direction is measured by Mott scattering from a thin gold foil after accelerating the electrons to an energy of $100 \mathrm{keV}$. In cases where the emitted beam is in part longitudinally polarized a Wien filter (crossed electric and magnetic fields) is used for turning the polarization into a purely transverse one.

For measuring the electron energy distribution a retarding-field analyser is used. A Faraday cup, which can be moved out of the beam path during

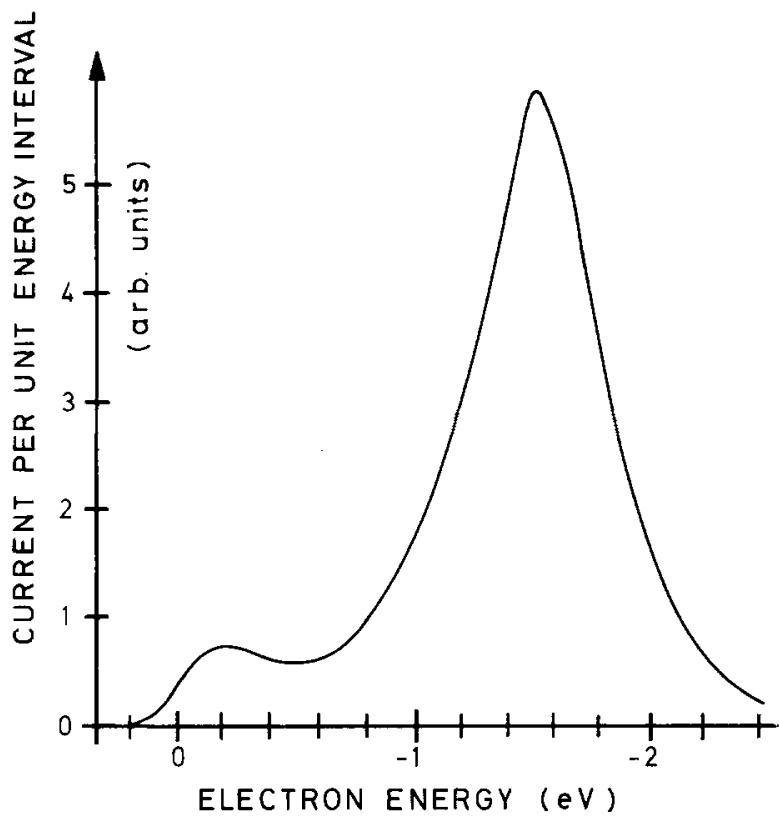

Fig. 2. Differential energy distribution of electrons from a W-EuS emitter which was annealed at a temperature of $910 \mathrm{~K}$. The zero point of the energy scale corresponds to the Fermi level of tungsten and was determined from the left shoulder of a distribution of electrons from an uncoated tungsten tip. The energy resolution of the retarding-field system is estimated to be about $150 \mathrm{meV}$. The right-side peak of the distribution corresponds to electrons which have suffered energy losses and lie energetically near the bottom of the EuS conduction band when they are emitted into the vacuum. In accordance with this view is the observation that the separation of the two peaks increases with both EuS-layer thickness and field strength. When a two-peak distribution is observed the spin polarization is typically 0.6 to 0.8

polarization measurements, is mounted right behind the probe hole of the screen [6]. The current to the Faraday cup is measured with an electrometer operated in the feedback mode to minimize the voltage drop across the input resistance. The retarding voltage is applied to the electrometer and increased in small steps synchronized to the channel advancement of a multichannel analyzer (MCA). The electrometer output signal is digitized and added to the memory of the corresponding MCA channel. In this way the current as a function of the retarding field, which gives the integral electron energy distribution, can be averaged over several sweeps. From this the differential energy distribution is obtained by a computer. Actually, a microprocessor is used to operate as an MCA, to generate the staircase function for the retarding voltage, to perform the differentiation, and to regulate the annealing current [9]. The differential energy 


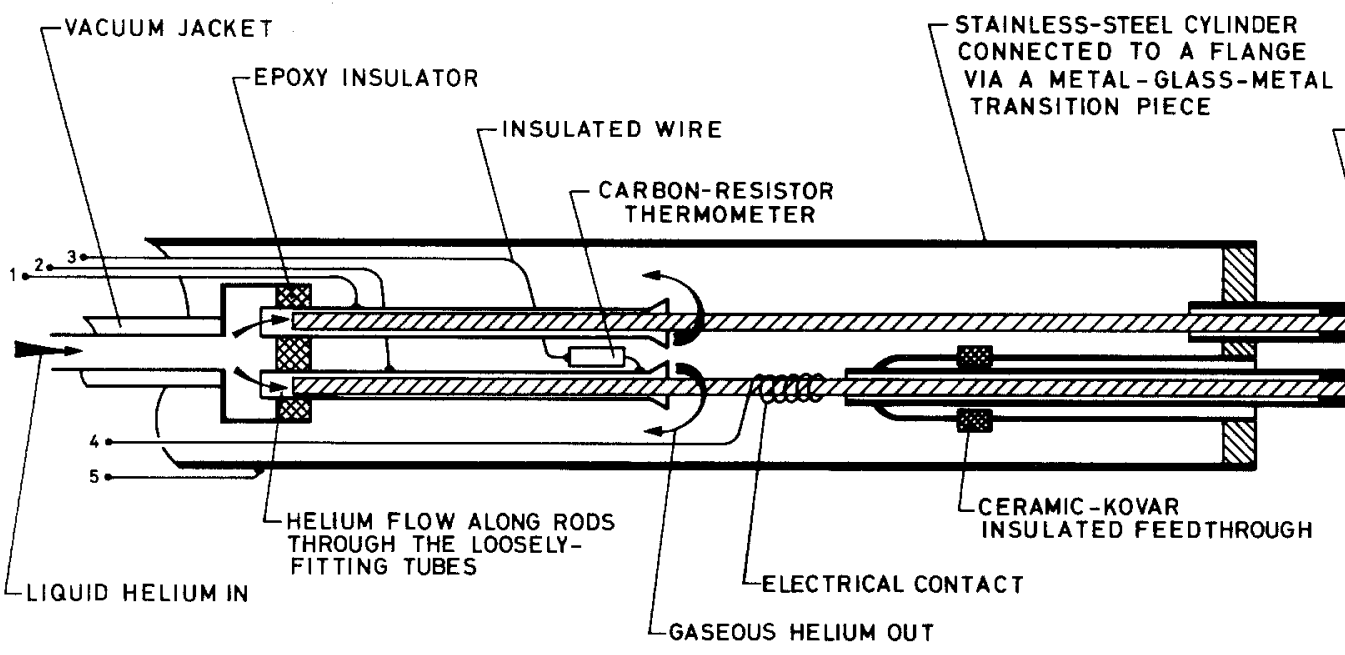

ig. 3. Cross section of emitter support. (Electrical connections: 1 and 2 - heating current through tungsten bow:2 and 3 - carbon thermometer; 4 and 5 - potential drop across bow; 5 - emitter potential of several kilovolts with respect to ground. The liquidhelium inlet pipe together with the tubes, carbon thermometer and wires No. 1, 2, and 3 form a plug which is inserted from the left. During operation the carbon thermometer measures the temperature some distance away from the tip; the deviation from the real tip temperature was determined in a separate test in which a second carbon thermometer was attached to the tungsten bow. The small overall size of this design was chosen to minimize liquid-helium consumption, which is 2 to $3 \mathrm{l} / \mathrm{h}$ ).

distribution is displayed on-line and also punched out on paper tape.

\section{Results}

The narrow energy distribution shown as Curve $a$ in Fig. 4 was obtained after annealing at the temperature of $840 \mathrm{~K}$ for 1s. This curve has to be interpreted as the convolution of the true distribution with the analyzer resolution function. For the latter a Gaussian can be assumed with a width of $150 \mathrm{meV}$, which is our experimental energy resolution. Following the evaluation procedure of Young and Kuyatt [10] we conclude that a good approximation of the true distribution is a curve with a width of $80 \mathrm{meV}$ and a shape as that of Curve $b$ in Fig. 4. Its steep high-energy shoulder suggests that it might be possible to produce an even more monoenergetic beam by simply using a filter lens for cutting off the low-energy tail.

When the energy distribution is narrow the field emission pattern consists only of a single spot. Annealing at a slightly higher temperature yields the typical pattern corresponding to emission from the EuS-coated (112) planes of tungsten, and also a broadened energy distribution similar to that of Fig. 2.

Together with this narrow energy distribution we measured a purely transverse spin polarization of
$0.85 \pm 0.10$ at an operating temperature of $9 \mathrm{~K}$. The transverse polarization implies that the direction of the spontaneous magnetization of the EuS layer lies tangential to the surface. Apparently, the emitting portion of the surface is a single Weiss domain. Whether the azimuthal direction of the polarization is determined only by a transverse magnetic stray field or also by crystal orientation is not yet known.

At a constant temperature of $9 \mathrm{~K}$ and a vacuum of $3 \times 10^{-11}$ Torr this emitter yields a stable current comparable to that of an uncoated tungsten tip. A total beam current of $10^{-8} \mathrm{~A}$ was measured. When the emitter temperature is increased, the emission current at constant extraction voltage decreases drastically and vanishes between 16 and $17 \mathrm{~K}$. This pronounced temperature dependence of the current was studied previously [4]; it is caused by the change of the spontaneous magnetization and the related conduction-band splitting below the Curie point.

\section{Interpretation}

It is assumed that the special condition of the W-EuS emitter described here is reached by an annealing procedure which allows europium and sulfur, deposited in the right stoichiometric proportions, to combine chemically to form EuS and to crystallize, 


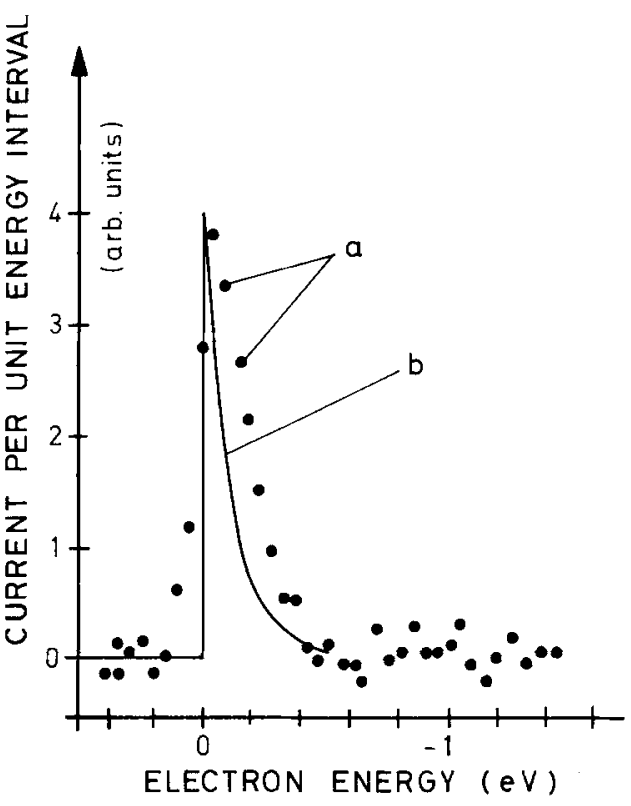

Fig. 4. (a) Narrow energy distribution measured after annealing the W-EuS emitter at $840 \mathrm{~K}$. - (b) Estimate of the true electron energy distribution. Curve $b$ folded with a Gaussian of $150 \mathrm{meV}$ half width gives a good approximation of Curve $a$

but which does not suffice for the development of an epitaxial interface at the W-EuS boundary. For the internal barrier to act as a spin filter it is necessary that the EuS be crystallized in order to become ferromagnetic and to have the conduction-band splitting, whereas epitaxy is not required.

A nearly perfect crystallization is suggested by the facts that the electrons traverse the EuS layer (which has a thickness of several hundred Angströms, $700 \AA$ before annealing as determined from the rate of EuS evaporation) without energy loss and that the measured Curie temperature lies between 16 to $17 \mathrm{~K}$. Impurities and crystal defects in the EuS layer would make electron energy losses more likely and also raise the Curie temperature [11]. Upon further annealing, the broad energy distributions similar to that of Fig. 2 are observed together with a (112) field emission pattern.

This leads us to assume that significant inelastic processes occur in scattering from dislocation which are introduced when the EuS crystal (fcc, lattice constant $a=5.97 \AA$ ) is epitaxially matched to the tungsten crystal (bcc, $a=3.16 \AA$ ).

The special annealing temperature of $840 \mathrm{~K}$ (for which an error of $\pm 50 \mathrm{~K}$ was estimated) is consistent with the observation of Schwob and Everett [12] who found that annealing of $\mathrm{EuS}$ at $500{ }^{\circ} \mathrm{C}$ leads to a perfectly crystallized sample.

\section{Application}

High spin-polarization, narrow energy width, a stable current and an extremely small beam emittance make this W-EuS emitter a unique polarized electron source. In Table 1 its performance characteristics are compared with those of competitive sources. For calculating the emittance $\varepsilon_{0}$ of the W-EuS emitter we assumed that electrons of about $3 \mathrm{eV}$ energy are emitted from a spot with an area of $A \sim 10^{-12} \mathrm{~cm}^{2}$ into a solid angle of $\Omega=2 \pi$ (approximation: $A \Omega \sim \pi^{2} \varepsilon_{0}^{2}$ ). The extended figure of merit, $M_{3}$, is introduced here in order to express the advantage of a small energy width. For polarization experiments with high energy resolution it is the most pertinent

Table 1. Performance parameters of low-energy polarized electron sources

\begin{tabular}{|c|c|c|c|c|c|c|}
\hline Source process & $\begin{array}{l}\text { Low-energy } \\
\text { mott scattering } \\
{[13]}\end{array}$ & $\begin{array}{l}\text { Fano effect } \\
\text { of Cesium } \\
{[14,2]}\end{array}$ & $\begin{array}{l}\text { Optically pumped } \\
\text { He discharge } \\
{[15]}\end{array}$ & $\begin{array}{l}\text { Two-photon } \\
\text { ionization } \\
\text { of Cs [16] }\end{array}$ & $\begin{array}{l}\text { Photo emission } \\
\text { from GaAs } \\
{[17]}\end{array}$ & $\begin{array}{l}\text { Field emission } \\
\text { from W-EuS }\end{array}$ \\
\hline Polarization $P$ & 0.2 & 0.65 & 0.3 & (goal 1.0) & 0.4 & 0.85 \\
\hline Current $I[\mathrm{~A}]$ & $3.5 \times 10^{-8}$ & $3 \times 10^{-9}$ & $5 \times 10^{-7}$ & (goal $10^{-7}$ ) & (goal $10^{-3}$ ) & $10^{-8}$ \\
\hline Emittance $\varepsilon_{0}[\mathrm{rad} \mathrm{cm}]$ & $2 \times 10^{-2}$ & $10^{-2}$ & $10^{-2}$ & $10^{-1}$ & $2 \times 10^{-3}$ & $8 \times 10^{-7}$ \\
\hline at energy $E_{0}[\mathrm{eV}]$ & $3 \times 10^{2}$ & $10^{3}$ & $5 \times 10^{2}$ & $3 \times 10^{-2}$ & 1 & 3 \\
\hline Energy width $\Delta E[\mathrm{eV}]$ & 0.6 & 2 & 0.5 & 0.5 & 0.2 & 0.1 \\
\hline $\begin{array}{l}\text { Customary figure of merit } \\
\qquad M_{1}=I P^{2}[\mathrm{~A}]\end{array}$ & $1.4 \times 10^{-9}$ & $1.3 \times 10^{-9}$ & $4.5 \times 10^{-8}$ & $\left(10^{-7}\right)$ & $\left(1.6 \times 10^{-4}\right)$ & $7.2 \times 10^{-9}$ \\
\hline $\begin{array}{l}\text { "Beam quality" }[13] \\
M_{2}=M_{1} / \varepsilon_{0}^{2} E_{0} \\
{\left[\mathrm{~A} / \mathrm{rad}^{2} \mathrm{~cm}^{2} \mathrm{eV}\right]}\end{array}$ & $1.2 \times 10^{-8}$ & $1.3 \times 10^{-8}$ & $9 \times 10^{-7}$ & $\left(3 \times 10^{-4}\right)$ & $(40)$ & $3.8 \times 10^{3}$ \\
\hline $\begin{array}{l}\text { Extended figure of merit } \\
M_{3}=M_{2} / \Delta E \\
{\left[\mathrm{~A} / \mathrm{rad}^{2} \mathrm{~cm}^{2} \mathrm{eV}^{2}\right]}\end{array}$ & $2.0 \times 10^{-8}$ & $6.5 \times 10^{-9}$ & $1.8 \times 10^{-6}$ & $\left(6 \times 10^{-4}\right)$ & $\left(2 \times 10^{2}\right)$ & $3.8 \times 10^{4}$ \\
\hline
\end{tabular}


quantity. All the sources listed in Table 1 operate with a weak or without a magnetic field and yield dc beams of low-energy electrons.

The transverse beam polarization (as obtained with this source in a weak or zero magnetic field) has the advantage that it can be measured by Mott scattering without prior polarization conversion. For polarization reversal a transverse magnetic field could be applied when the tip temperature goes below $T_{c}$, thus forcing the spontaneous magnetization into the desired direction. A longitudinal polarization can be obtained with a longitudinal magnetic field of several kilogauss at the tip.

We will employ this source in an experiment on the scattering of low-energy polarized electrons from polarized lithium atoms.

Acknowledgement. This work has been supported by the University of Bielefeld under Project No. 2854.

\section{References}

1. J.Kessler: Polarized Electrons (Springer, Berlin, Heidelberg, New York 1976)

2. M.S.Lubell: In Atomic Physics, Vol. 5, ed. by R.Marrus, M.Prior, and H.Shugart, (Plenum Press, New York, London 1977), pp. 325-373
3. N. Müller, W.Eckstein, W.Heiland, W.Zinn: Phys. Rev. Lett. 29, 1651-1654 (1972)

4. E. Kisker, G. Baum, A.H.Mahan, W.Raith, K.Schröder: Phys. Rev. Lett. 36, 982-985 (1976)

5. E. Kisker, A.H.Mahan, B.Reihl; 8th Int. Coll. Magnetic Thin Films, York, England (1976) Abstracts p. 45

6. E. Kisker, A.H. Mahan, B. Reihl: Physics Lett. A (in print)

7. D.A.Reed, W.R.Graham: Rev. Sci. Instrum. 43, 1365-1367 (1972)

8. Purchased from Field Electron and Ion Source Specialists Company McMinnville, Oregon 97128, USA

9. E. Kisker: J. Phys. E Scient. Instrum., to be published

10. R.D. Young, C. E. Kuyatt: Rev. Sci. Instrum. 39, 1477-1480 (1968)

11. E. Bayer, W.Zinn: Z. angew. Physik 32, 83-88 (1971)

12. P.K.Schwob, G.E.Everett: J. Phys. 32, C1 1066-1067 (1971)

13. Reference 1, Chapter 7.3

14. G.Baum, M.S.Lubell, W. Raith: Bull. Am. Phys. Soc. 16, $586(1971)$

15. P.J.Keliher, R.E.Gleason, G.K. Walters: Phys. Rev. A.11, $1279-1285$ (1975)

16. E.H.A.Granneman, M.Klewer, K.J.Nygaard, M.J.Van der Wiel: J. Phys. B 9, L 87-L 91 (1976);

E.H.A.Granneman: Thesis, Univ. Amsterdam, 1976. For determining the emittance of this source listed in Table I it was assumed that electrons of $0.03 \mathrm{eV}$ energy are emitted from an area of $10^{-2} \mathrm{~cm}^{2}$ in all directions

17. D. T. Pierce, F. Meier, P.Zürcher: Appl. Phys. Lett. 26, 670-672 (1975);

D.T.Pierce, F. Meier: Phys. Rev. B 13, 5484-5500 (1976) 